\title{
SUPPLEMENTAL INFORMATION \\ Investigation of the spatial distribution of methane sources in the Greater Toronto Area using mobile gas monitoring systems
}

Sebastien Ars ${ }^{*}, 1,2$, Felix Vogel ${ }^{1}$, Colin Arrowsmith², Sajjan Heerah' ${ }^{3}$ Emily Knuckey ${ }^{2}$, Juliette Lavoie ${ }^{2}$, Christopher Lee², Nasrin Mostafavi Pak2 , Jaden L. Phillips², and Debra Wunch²

${ }^{1}$ Environment and Climate Change Canada, Climate Research Division, Toronto, Ontario, Canada

2 University of Toronto, Department of Physics, Toronto, Ontario, Canada

${ }^{3}$ Los Alamos National Laboratory, Los Alamos, NM, USA

*Corresponding author, Email: sebastien.ars@canada.ca

NUMBER OF PAGES: 16

NUMBER OF TABLES: 3

NUMBER OF FIGURES: 10

This supplementary information contains information about 1) the location of the five studied sources, 2) pictures of the two mobile platforms, 3) the estimation of the background mixing ratios, 4) the comparison of methane mixing ratios measured by the instruments of both platforms, 5 ) the triangulation of the source locations, 6) the inverse modeling approach, 7) the surveys realized in the GTA between May 2018 and August 2019 with both mobile analytical platforms, 8) the distribution of the number of enhancements per visit in the GTA, 9) the Gaussian Cluster Mixture Analysis, 10) the list of the datasets used in the inverse modeling approach. 


\section{TABLE OF CONTENTS}

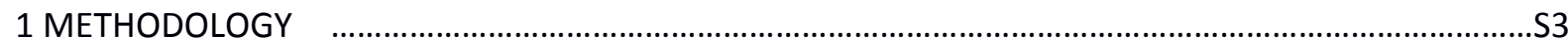

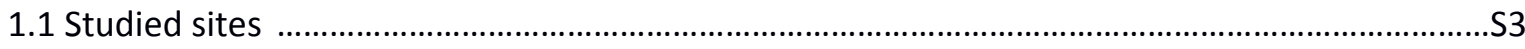

1.2 Presentation of the mobile platforms

1.3 Estimation of the methane background

1.4 Example of observed enhancements

1.5 Comparison of the two instruments

1.6 Triangulation of the source location

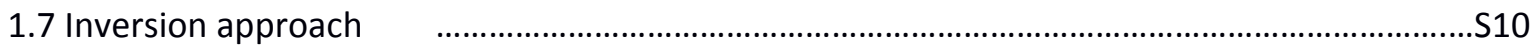

2 RESULTS

2.1 Surveys throughout the GTA

2.2 Distribution of the number of enhancements per visit ............................................................

2.3 Impact of the area criterion on the classification of the enhancements

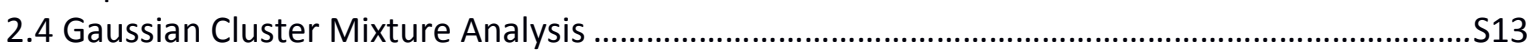

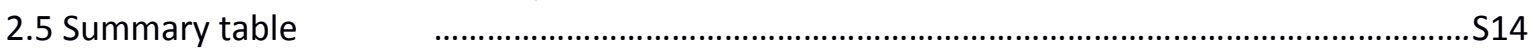

2.6 Datasets used in the inverse modeling approach

3 REFERENCES

\section{LIST OF FIGURES}

Figure S1: Location in the GTA of the five studied sources: the Keele Valley and Thackeray landfills, the Parkway and the Maple compressor stations, and the Keating Channel.

Figure S2: Presentation of the mobile platforms.

Figure S3: Illustration of the estimation of the background mixing ratios in a two-step process using

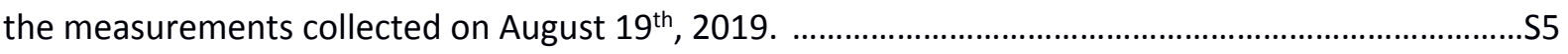

Figure S4: Example of enhancements observed during the August 9, 2019 survey .............................S6 Figure S5: Comparison of methane mixing ratios measured by the instruments of both platforms during the survey of April 24th, 2019. ............................................................................................

Figure S6: Comparison of the enhancements observed during the surveys with simultaneous

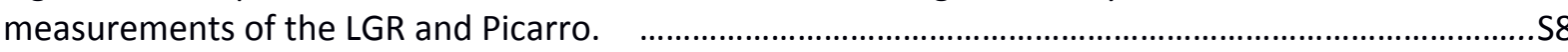

Figure S7: Example of the triangulation of the source locations for the Parkway natural gas compressor station.

Figure S8: Distribution of the number of enhancements per visit.

Figure S9: Distribution of the number of enhancements across small, medium and large categories when the only selection criterion is the maximum amplitude and when the area is also taken into account.

Figure S10: Map locating major sources of methane from downtown Toronto with the Gaussian Mixture Cluster Analysis.

\section{LIST OF TABLES}

Table S1: Summary of the surveys realized in the GTA between May 2018 and August 2019 with both mobile analytical platforms.

Table S2: Summary table of the emission rates estimated for different methane sources. $\quad$..........S14

Table S3: List of the datasets used in the inverse modeling approach for the different sites. .........S15 


\subsection{Studied sites}

Figure S1 presents the location of the two landfills, the two compressor stations, and the waterway whose emissions are estimated in this study.

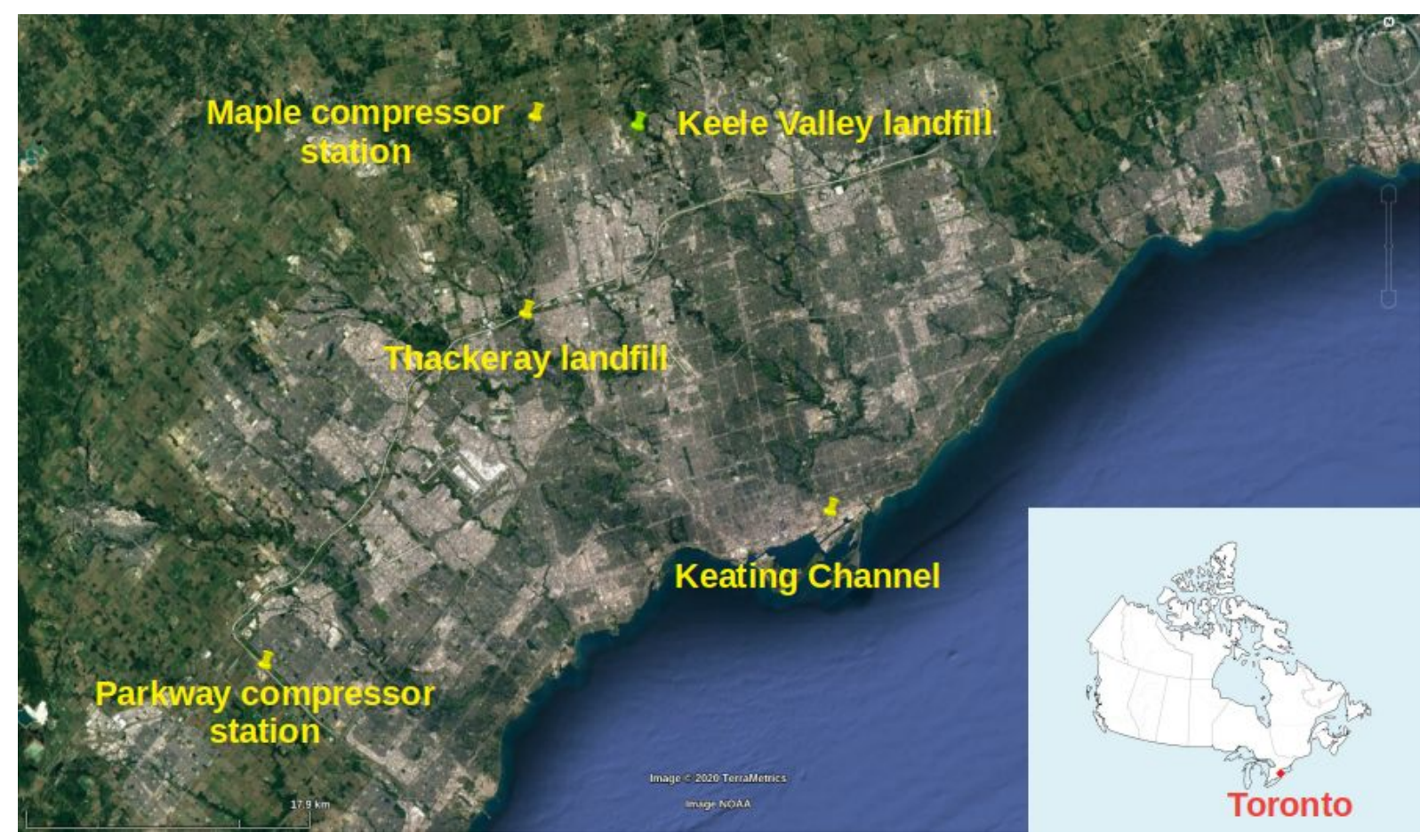

Figure S1: Location in the GTA of the five studied sources: the Keele Valley and Thackeray landfills, the Parkway and the Maple compressor stations, and the Keating Channel.

\subsection{Presentation of the two mobile platforms}

Figure S2 shows pictures of the two mobile platforms deployed in the GTA to investigate methane emissions.

Mole fractions of $\mathrm{CH}_{4}, \mathrm{CO}_{2}$ and $\mathrm{H}_{2} \mathrm{O}$ are measured simultaneously using an ultraportable multi-gas carbon emissions analyzer developed by Los Gatos Research (Mountain View, California, USA) embedded in a trailer for the bicycle-based platform. The car-based platform contains a G1301 analyzer developed by Picarro (Santa Clara, California, USA). The ultraportable multi-gas carbon emissions analyzer has a stated precision of $3 \mathrm{ppb}, 0.4 \mathrm{ppm}$, and $60 \mathrm{ppm}$ for $\mathrm{CH}_{4}, \mathrm{CO}_{2}$ and $\mathrm{H}_{2} \mathrm{O}$ over a $1 \mathrm{~s}$ integration period, respectively. Both $\mathrm{CH}_{4}$ and $\mathrm{CO}_{2}$ mixing ratios collected with this analyzer since July $13^{\text {th }}, 2018$, are calibrated against two tanks calibrated on the WMOX2004A scale for $\mathrm{CH}_{4}$ and the WMOX2007 scale for $\mathrm{CO}_{2}$. Data collected before July 13, 2018 are uncalibrated. The G1301 analyzer is based on cavity ring-down spectroscopy (CRDS) and its stated precision is $1 \mathrm{ppb}, 0.2 \mathrm{ppm}$ and 100 ppm over a $5 \mathrm{~s}$ integration period for $\mathrm{CH}_{4}$ and $\mathrm{CO}_{2}$, respectively. Both $\mathrm{CH}_{4}$ and $\mathrm{CO}_{2}$ mixing ratios collected with this analyzer are calibrated against the same two tanks. Data collected with both instruments were water-corrected, detailed comparisons between the two instruments are provided in the Supporting Information. The sampling inlet was mounted on a mast $2 \mathrm{~m}$ above the ground for the bicycle-based platform and at $2.5 \mathrm{~m}$ for the car-based system. In previous mobile surveys across 
US cities, the sampling inlet was located closer to the ground. The impact of the sampling line height was studied by simultaneously sampling one instrument close to the ground and the other instrument at $2.5 \mathrm{~m}$ during four surveys in April 2019. These preliminary tests showed that both instruments were able to detect most methane enhancements, but their maximum amplitude was on average between 25 and $37 \%$ lower when sampled with the higher inlet than when sampled close to the ground. The air sampled close to the ground also presents much higher enhancements of carbon dioxide mixing ratios than at $2.5 \mathrm{~m}$ because of the proximity of other cars' exhaust pipes, especially in traffic jams. The temporal offsets between the moment when the air is sampled by the inlet and when it reaches the cavity of the analyzer are carefully calculated in both platforms by injecting air with enhanced mixing ratio into the inlet and timing the response of the instrument.

On the bicycle-based platform, we collect wind data using a 220WX weather station (Airmar, Milford, New Hampshire, USA) and that automatically corrects wind speed and direction using an embedded GPS sensor. For the car-based platform, we use two different sensors for the wind measurements. From August 2018 to the end of October 2018, we used an AIO 2 sonic weather sensor (Met One Instruments, Grants Pass, Oregon, USA) and a 220WX weather station after November 2018. A correction is applied to the wind data collected with the AIO 2 for both direction and speed to remove the impact of the vehicle's motion. The stated accuracies for the wind speed and direction for both sensors are $0.5 \mathrm{~m} / \mathrm{s}$ and $5^{\circ}$, respectively. The platform coordinates were recorded using the GPS in the 220WX weather station when available or the GPS in a Garmin watch. The stated accuracy of each GPS receiver is $3 \mathrm{~m}$ but larger errors have been observed in the downtown area where the roads are surrounded by tall buildings, limiting GPS reception.

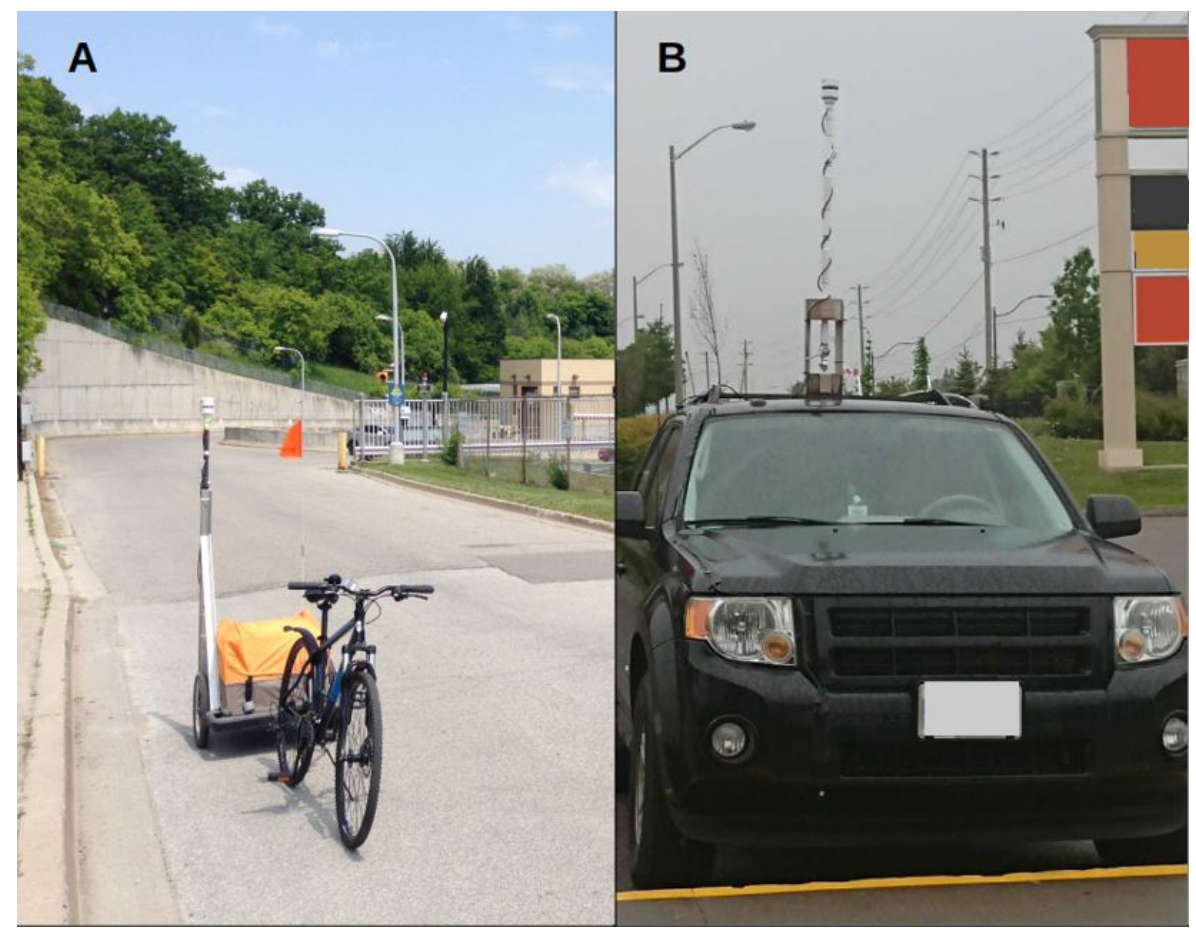

Figure S2: Presentation of the two mobile platforms: (A) the bicycle-based platform, and (B) the car-based platform.

\subsection{Estimation of the methane background}

To take into account spatiotemporal fluctuations in methane mixing ratios, the background is estimated using a two-step process illustrated in Figure S3. A first estimate of the background is 
derived from the fifth percentile of a moving window $(1,2)$ whose length was optimized for each analytical platform based on their different motion speed. When the moving window encounters a wide enhancement, the estimated background produces an irregular increase represented in red in Figure S3 B. This background irregularity is corrected in a second step by looking at the standard deviation of this first estimate of the background which increases during those events (Figure S3 C). The irregular portions of the background with a standard deviation exceeding a certain limit are filtered and replaced by interpolated values (Figure S3 D).
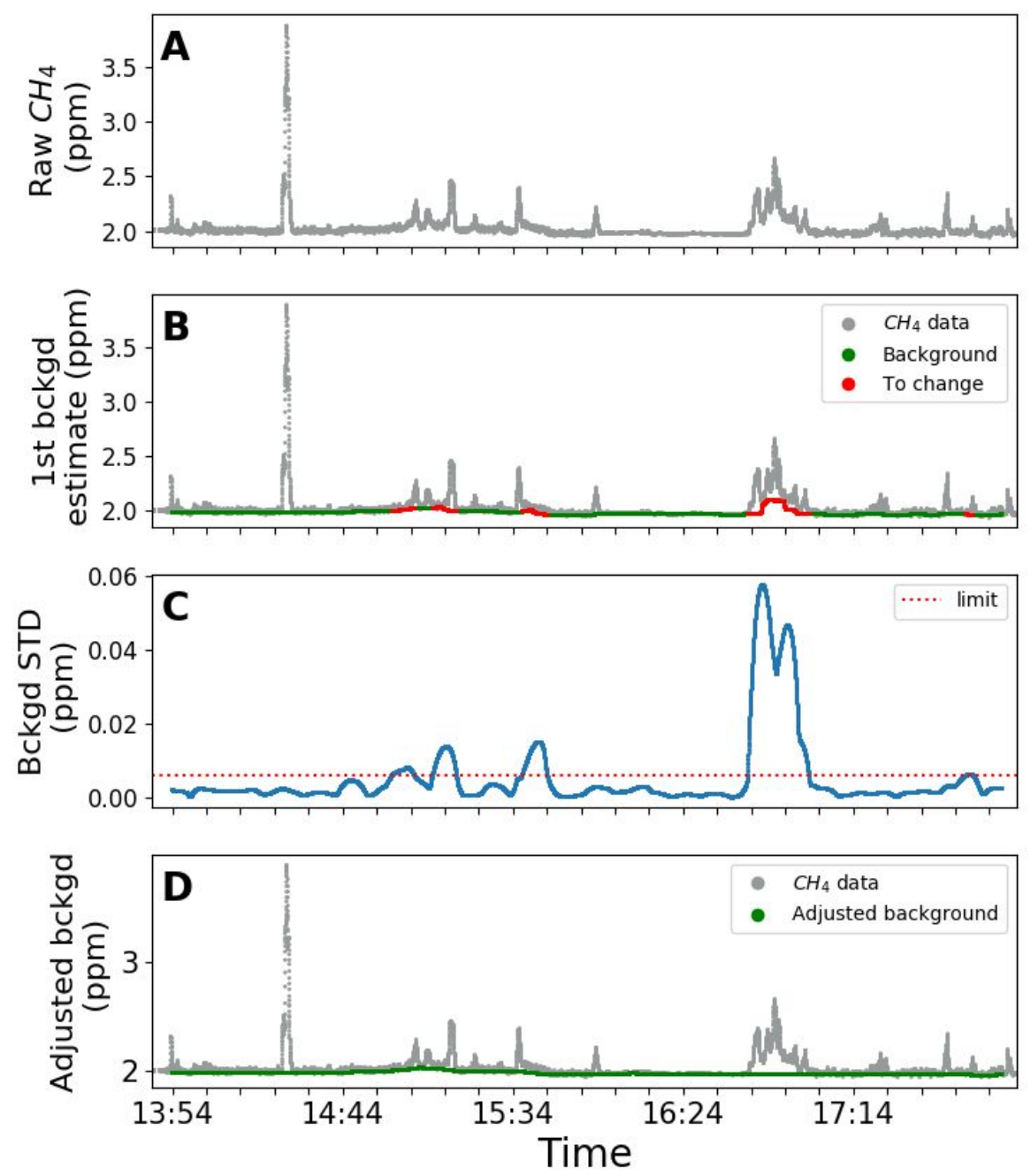

Figure S3: Illustration of the estimation of the background mixing ratios in a two-step process using the measurements collected on August 19th 2019 . (A) Methane mixing ratios measured during this survey. (B) First estimate of the background as the fifth percentile of a moving window represented in green. Irregularities in this first estimate are coloured in red. (C) Standard deviation of the background first estimate. (D) Adjusted background in green.

\section{$97 \quad 1.4$ Example of observed enhancements}

98 Figure S4 presents the example of measurements collected on August 9, 2019 after background 99 subtraction. The enhancements of methane mixing ratios are classified in to three categories 


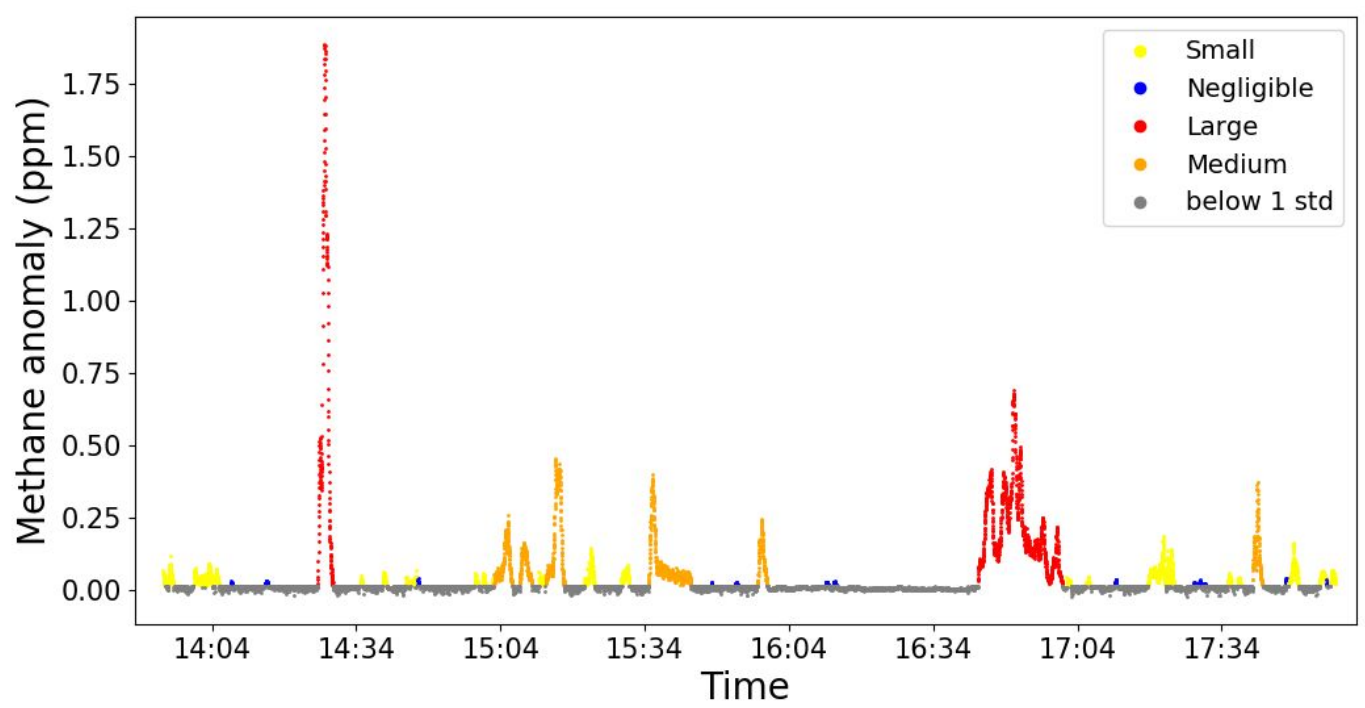

Figure S4: Example of enhancements observed during the August 9, 2019 survey. Small, medium and large enhancements are coloured in yellow, orange and red, respectively.

\subsection{Comparison of the two instruments}

103 Several surveys were performed with analyzers in the car-based platform: one survey on November, 1042018 with both instruments sampling at $2.5 \mathrm{~m}$ above the ground and four surveys on April, 2019 with 105 the Picarro sampling close to the ground and the LGR sampling at $2.5 \mathrm{~m}$.

106 Figure S5 (left panel) shows the time series of methane mole fractions measured on April 25th 2019 107 by the LGR analyzer in red and the Picarro analyzer in gray. Figure S5 (right panel) shows the 108 correlation between these two sets of measurements. The slope obtained with the raw data is 0.82 109 and increases to 1.01 when the Picarro analyzer data are averaged over a period of 20 seconds, 110 compensating for different residence times in the inlet tubing, due to the instrument specific flow 111 rates. 

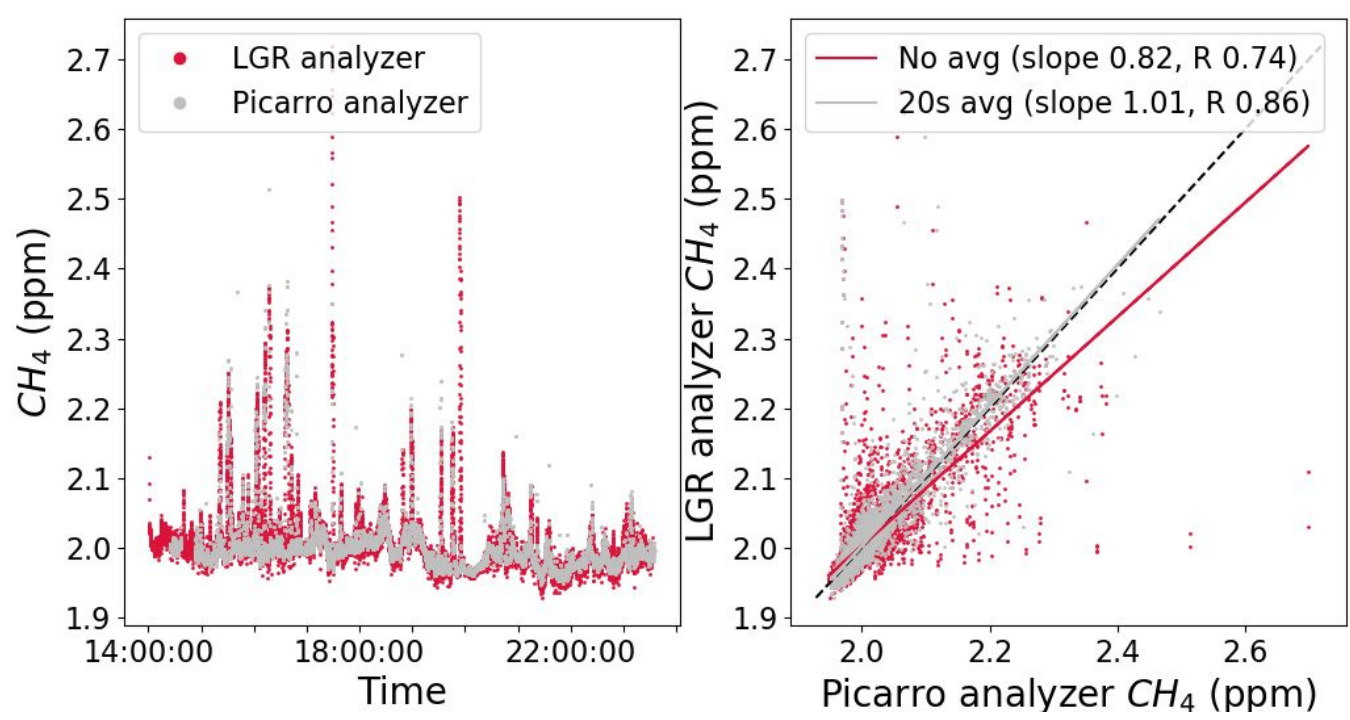

Figure S5: Comparison of methane mixing ratios measured by the instruments of both platforms during the survey of April 25th, 2019. The graph on the left shows the time series of methane mole fractions measured by the ultraportable multi-gas carbon emissions analyzer (LGR) in red and the G1301 analyzer (Picarro) in gray. The graph on the right presents the correlation between the methane mole fractions measured by both instruments, the scatter plot in red is obtained with the raw Picarro analyzer data whereas the scatter plot in gray shows Picarro analyzer data averaged over a period of 20 seconds. The LGR data stay the same and are not averaged.

We compared the height and area of the enhancements observed when both analyzers were sampling simultaneously. Figure S6 A shows the comparison between the enhancements height measured when both instruments were sampling at $2.5 \mathrm{~m}$ and Figure S6 B shows the comparison of their area during the same survey. The Picarro seems to measure enhancements with a maximum amplitude about $30 \%$ lower than the LGR, but the areas measured by both instruments seem very consistent. Figure S6 C shows the comparison between the enhancements height measured when the LGR was sampling at $2.5 \mathrm{~m}$ and the Picarro was sampling close to the ground. Figure S6 D shows the comparison of the enhancements area during the same surveys. The Picarro, which was measuring close to the ground, shows enhancements with a maximum amplitude about $50 \%$ higher than the LGR, which was sampling at $2.5 \mathrm{~m}$. The areas measured by both instruments in these conditions remain very consistent and therefore the enhancements area should be considered a more reliable measurements of methane enhancements. Based on this results, we estimate that the bicycle-based platform (which is equipped with a LGR and has a high sampling inlet) should measure enhancements with a maximum amplitude about $50 \%$ lower than the setup used in the von Fischer et al. and Weller et al. approaches (which is equipped with a Picarro sampling close to the ground). As for the car-based platform (which is equipped with a Picarro sampling at $2.5 \mathrm{~m}$ ), we estimate that it should measure enhancements with a maximum amplitude about $80 \%$ lower than the setup used in the von Fischer et al. and Weller et al. approaches. 

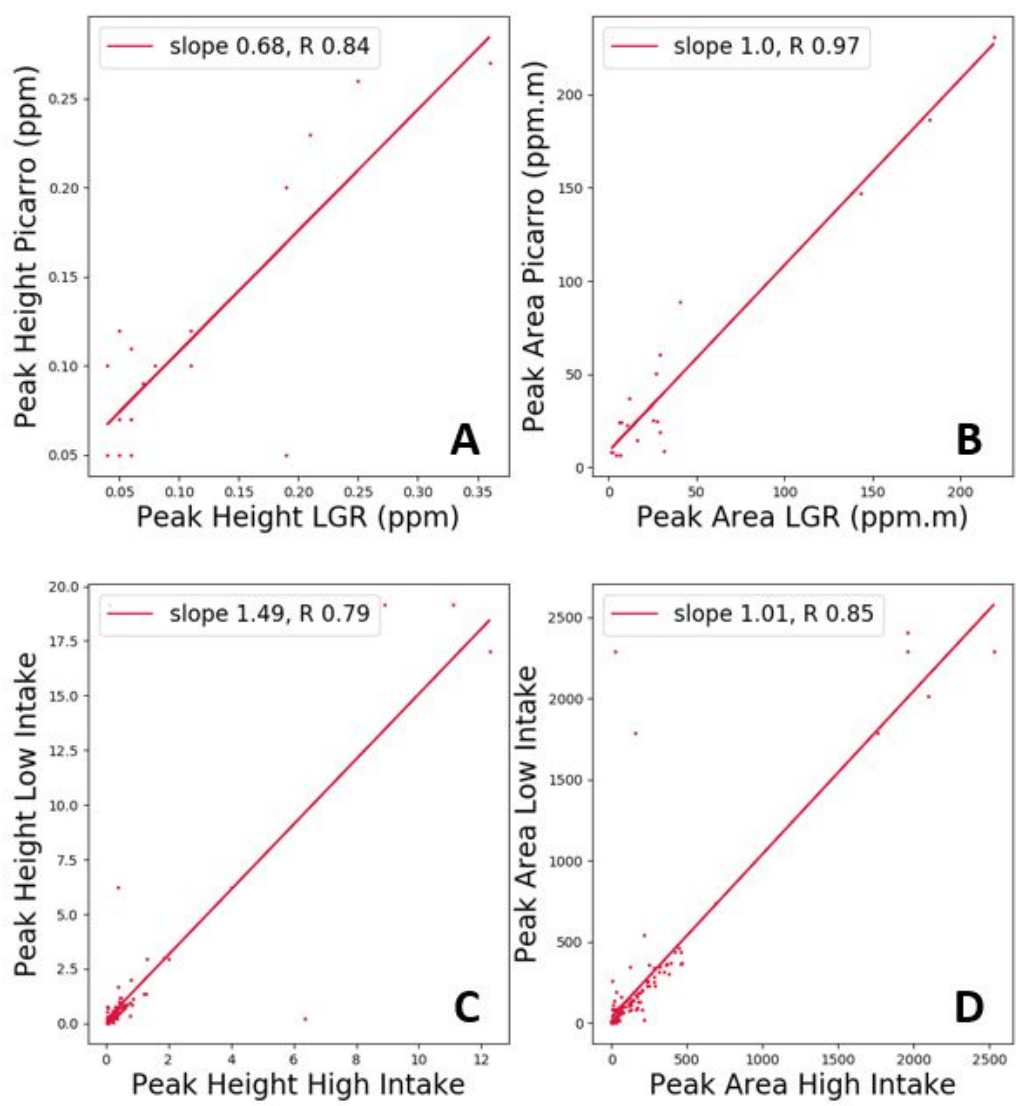

144 Figure S6: Comparison of the enhancements observed during the surveys with simultaneous 145 measurements of the LGR and Picarro: (A) The maximum enhancement heights when both the LGR 146 and Picarro sample at $2.5 \mathrm{~m}$ above the ground. (B) The enhancement areas when both instruments 147 sample at $2.5 \mathrm{~m}$ above the ground. (C) The maximum enhancement heights when the LGR samples at $1482.5 \mathrm{~m}$ above the ground and the Picarro samples close to the ground. (D) The enhancement areas 149 when the LGR samples at $2.5 \mathrm{~m}$ above the ground and the Picarro samples close to the ground.

\subsection{Triangulation of the source location}

151 The number of sources within a site is deduced by identifying the number of local extrema in the 152 plume under different wind conditions. Their location is estimated by using the prevailing wind 153 direction and triangulating these extrema as illustrated in Figure S7. 


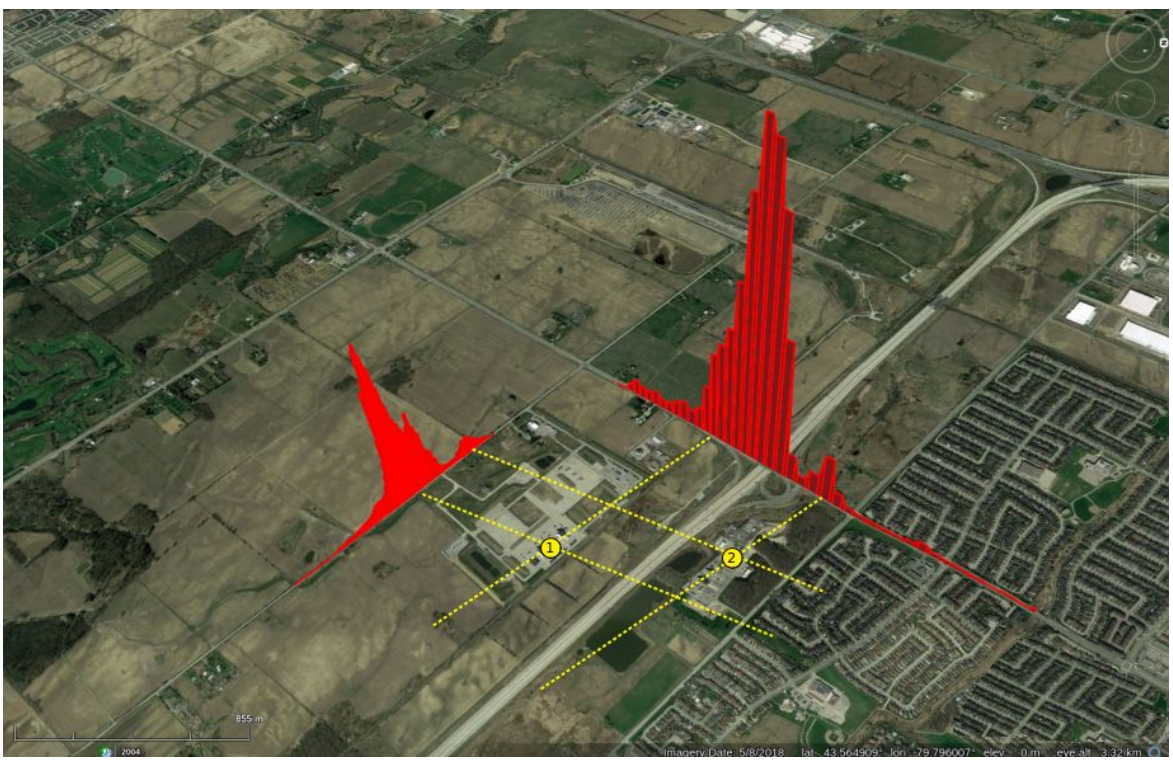

156

157 Figure S7: Example of the triangulation of the source locations for the Parkway natural gas 158 compressor station. Two transects of the emission plume measured under different wind conditions, 159 represented in red, are used to estimate the location of the main sources of the site. 


\subsection{Inversion approach}

161 The inversion method used in this study is based on a statistical inversion coupled with a Gaussian 162 model and is described in more details in Ars et al. (2017) (3). This is a Bayesian approach which aims 163 at updating a prior estimate of the emissions $f^{\text {b }}$, derived from our best available knowledge, with 164 statistical information from observations $\mathbf{p}$. For the Keele Valley and Thackeray landfills, we used the 165 estimates reported in the FLAME-GTA inventory as prior estimates of the emissions; these prior 166 emissions were equally distributed between the two identified sources. For the other sites, we 167 arbitrarily chose the prior estimate of the emissions. The uncertainty in these prior estimates were 168 set to $80 \%$ of the prior values and were included in the covariance matrix, B. Similar to Ars et al. 169 (2017) (3), the observations used in this inversion are the integrated areas under the enhancements. 170 When there are several sources of methane within a given site, plumes are divided into several slices 171 over space and the observations used in the inversion are the area under each slice. The relationship 172 between these observations and the emission rates relies on the knowledge of both the location of 173 the sources and the atmospheric transport. The relationship is expressed mathematically by the 174 observation operator, $\mathbf{H}$, and is determined with the Gaussian model. In practice, this observation 175 operator is computed by simulating the plume of each source separately with the atmospheric 176 conditions observed during each transect, using a unitary emission rate. Then we calculate the area 177 under the different slices of the modeled plume to compute the observation operator. The 178 uncertainties associated with the observation and the observation operator are characterized by the 179 covariance matrix $\mathbf{R}$. The posterior estimate of the emissions $f^{a}$ can be obtained from the equation:

$f^{a}=f^{b}+B H^{\top}\left(R+H B H^{\top}\right)^{-1}\left(p-H f^{b}\right)$

181

182 183 184 185

With this inversion approach, all the transects observed during each survey are used to estimate the emissions from a given site and thus reduces the uncertainty in our estimate.

\section{RESULTS}

\subsection{Surveys throughout the GTA}

Table S1 presents the number of surveys and the distance covered with each analytical platform from May 2018 to August 2019. 


\begin{tabular}{|c|c|c|c|c|}
\hline & & Total & Bike & Car \\
\hline \multirow{6}{*}{2018} & Distance (km) & 3,703 & 732 & 2,971 \\
\hline & Number of surveys & 41 & 27 & 14 \\
\hline & $\begin{array}{c}\text { Number of daytime } \\
\text { surveys }\end{array}$ & 30 & 27 & 3 \\
\hline & $\begin{array}{c}\text { Number of nighttime } \\
\text { surveys }\end{array}$ & 11 & 0 & 11 \\
\hline & Average time coverage & - & $3: 27$ & $6: 30$ \\
\hline & $\begin{array}{l}\text { Average vehicle speed } \\
\qquad(\mathrm{km} / \mathrm{h})\end{array}$ & - & 11 & 40 \\
\hline \multirow{6}{*}{2019} & Distance (km) & 2,741 & 1,007 & 1,734 \\
\hline & Number of surveys & 36 & 29 & 7 \\
\hline & $\begin{array}{c}\text { Number of daytime } \\
\text { surveys }\end{array}$ & 33 & 29 & 4 \\
\hline & $\begin{array}{c}\text { Number of nighttime } \\
\text { surveys }\end{array}$ & 3 & 0 & 3 \\
\hline & Average time coverage & - & $3: 35$ & $6: 40$ \\
\hline & $\begin{array}{l}\text { Average vehicle speed } \\
\qquad(\mathrm{km} / \mathrm{h})\end{array}$ & - & 11 & 40 \\
\hline
\end{tabular}

Table S1: Summary of the surveys realized in the GTA between May 2018 and August 2019 with both mobile analytical platforms.

\subsection{Distribution of the number of enhancements per visit}

190 Figure $\mathrm{S} 8$ shows the distribution of the number of enhancements observed per $1 \mathrm{~km} \times 1 \mathrm{~km}$ grid cell. 191 Downtown Toronto has a larger number of enhancements per visit than other parts of the GTA. 


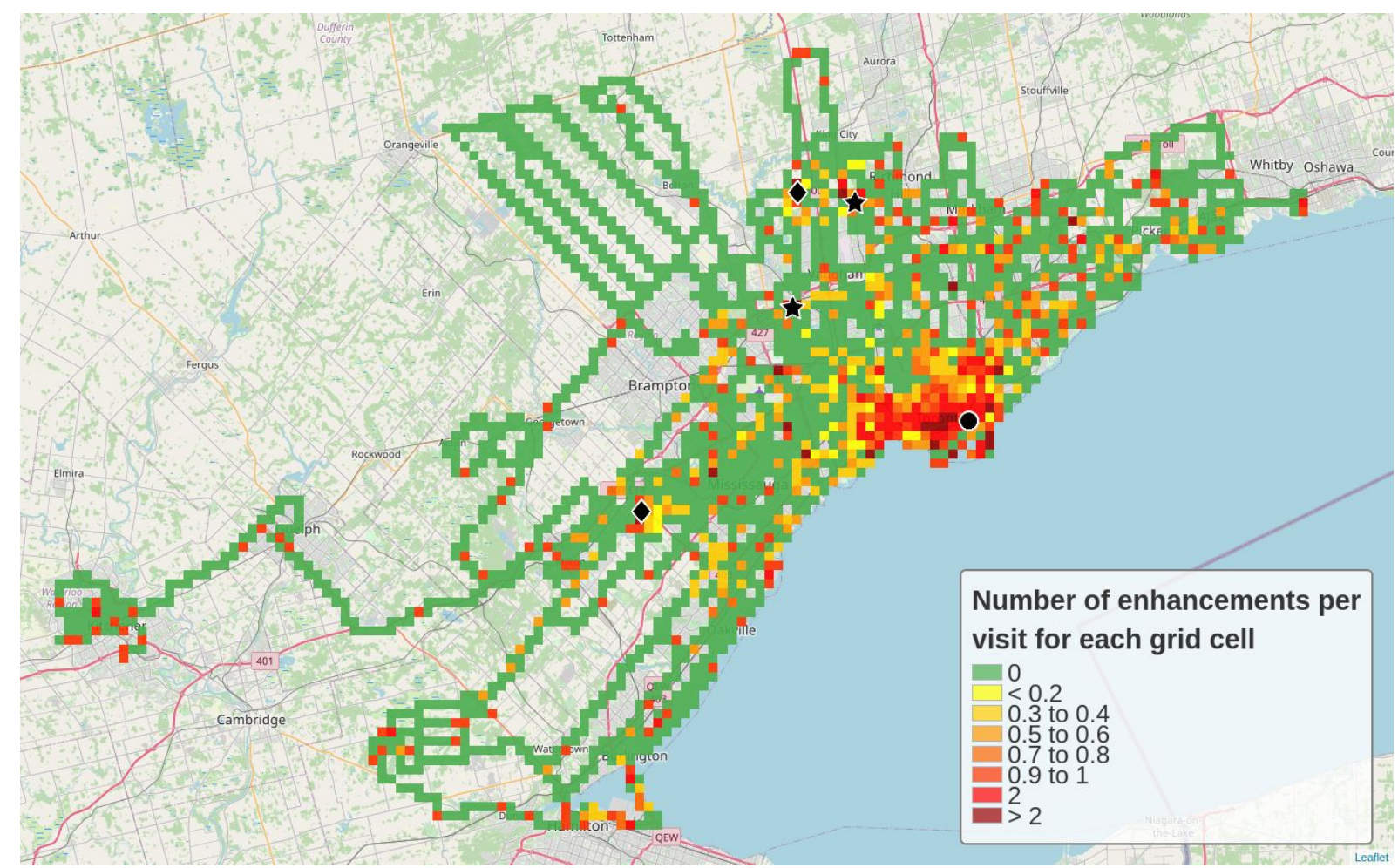

Figure S8: Distribution of the number of enhancements per visit for each grid cell. Boxes are coloured from yellow to red, yellow indicating an area with low number of enhancements per survey and grid cells coloured in dark red correspond to area with more than two enhancements per survey. Areas with no observed enhancement are coloured in green. The star, diamond and circle icons locate landfills, compressor stations, and the Keating Channel, respectively.

\subsection{Impact of the area criterion on the classification of the enhancements}

In this study, the classification of an enhancement across small, medium and large categories is based on two criteria: its maximum amplitude and its area. In previous studies, only the maximum amplitude was considered. The area criterion has been implemented to better account for large plumes whose source is distant from the measurement location. Such plumes usually have a relatively low maximum amplitude because of atmospheric dispersion and are classified into lower categories even though they are from sources with larger methane emissions. Figure S9 presents the difference in the distribution of the number of enhancements when we only take the maximum amplitude of the enhancements into account and when we also include their area. The implementation of the area criterion does not influence the distribution of the enhancements. Only $1 \%$ of the total observed enhancements are selected with the area criteria and considered as large plumes. 


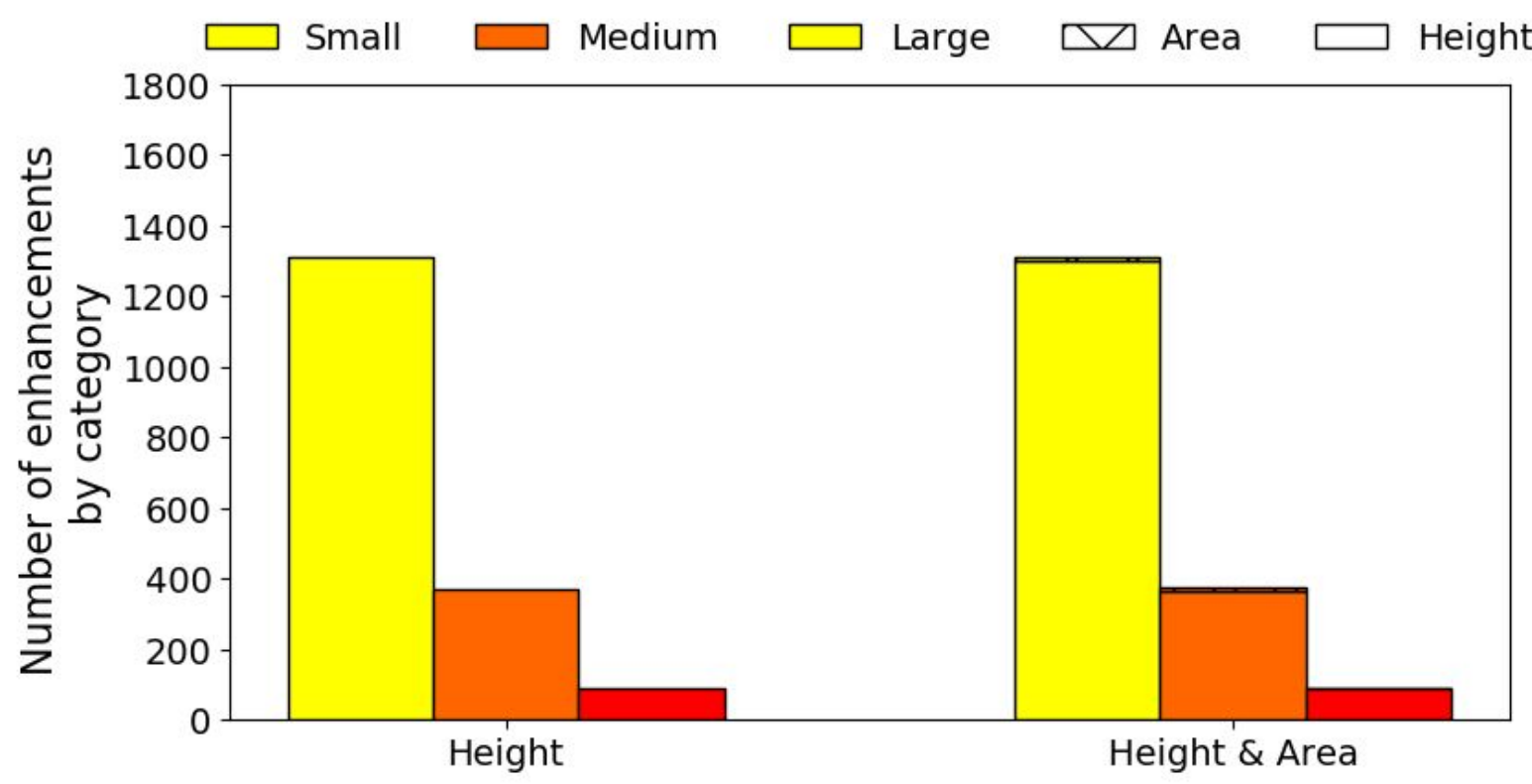

Figure S9: Distribution of the number of enhancements across small, medium and large categories when the only selection criterion is the maximum amplitude and when the area is also taken into account.

\subsection{Gaussian Mixture Cluster Analysis}

We have used a complementary machine learning tool to identify spatial clusters of methane enhancements. This method, called a Gaussian Mixture Cluster Analysis (GMCA) (4), is applied to the medium and large enhancements measured in the downtown area throughout the measurement period. Each enhancement location is shifted by the surface winds at the time of the measurement prior to applying the cluster analysis. The GMCA optimizes a model with N spatially Gaussian clusters, and the median location of each cluster can be interpreted as a potential methane emission source (https://bitbucket.org/wunchlab/gaussianclusters/).

The algorithm requires a minimum of 21 clusters to correctly locate four out of six of the methane emitters inventoried in the area. Beyond 31 clusters, the system starts to generate unrealistically small groups of fewer than five enhancements which reduce our confidence in the existence of a source corresponding to these small clusters. Therefore, we assume that the number of large sources of methane in downtown Toronto ranges between 21 and 31.

The output of the GMCA with 27 clusters is presented in Figure S10, representing potential sources of methane by the red icons. The locations of inventoried methane emitters are labeled with blue icons and show good agreement with most of the Gaussian cluster centres.

The GMCA is also able to locate unreported sources of methane. After further field investigations, some of these sources have been identified as closed landfills whose emissions are no longer reported (2 sources), and ponds or channels where organic matter accumulates and undergoes anaerobic decomposition such as the Keating Channel (7 sources). Other locations (6 sources) cannot be attributed to any of these types of sources, and thus further investigation is required using isotopic measurements, $\mathrm{VOC}$ measurements, and $\mathrm{CO}: \mathrm{CO}_{2}$ ratio analysis to identify the origin of the enhancements.

One limitation of the GMCA is that the algorithm can combine enhancements from separate sources. As a result, the analysis will tend to underestimate the total number of sources but will provide a good approximation of their mean location. More observations will improve the results of the GMCA. 


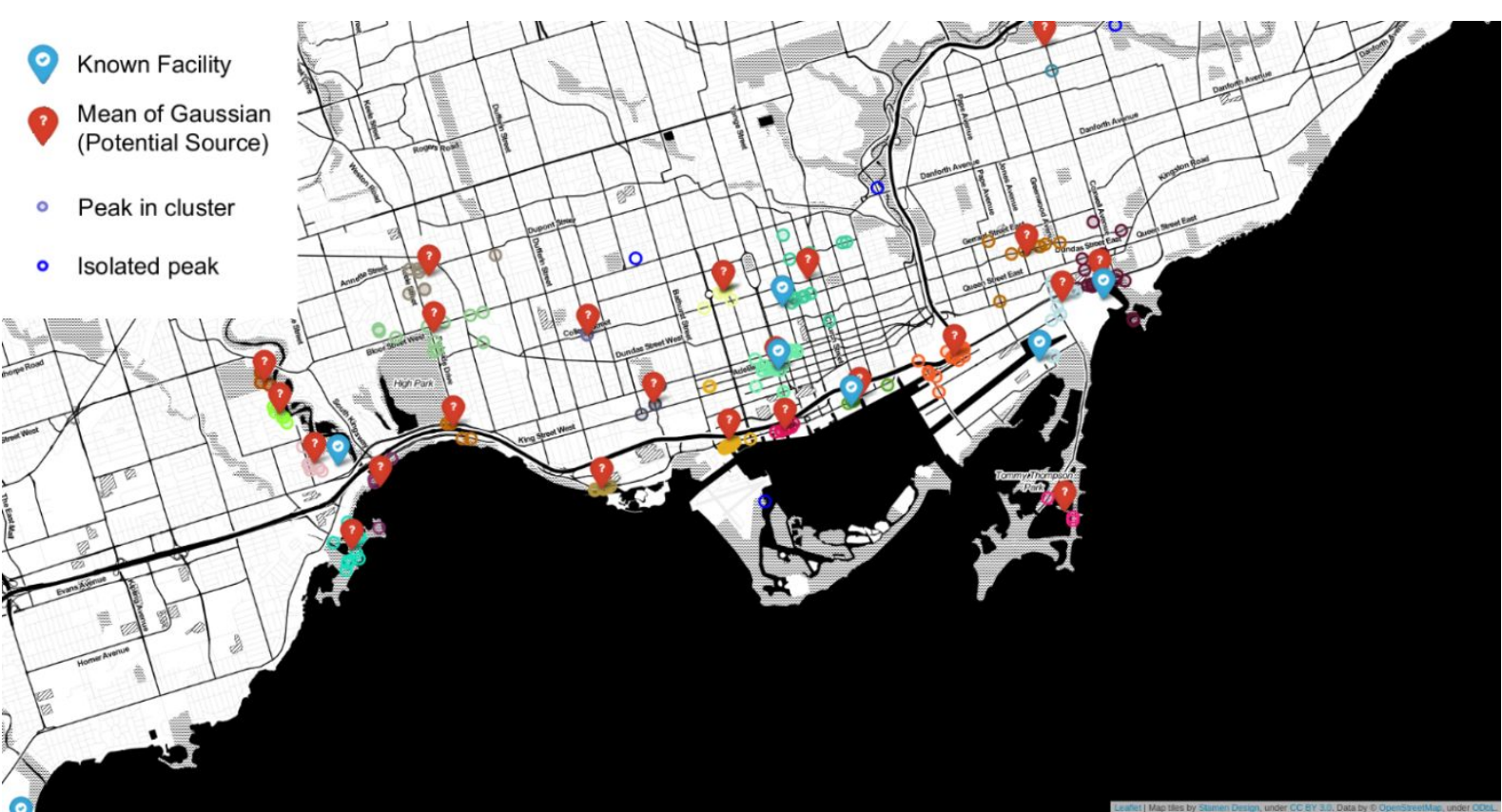

\begin{tabular}{|c|c|c|c|c|}
\hline & & Range of emissions (kg/d) & $\begin{array}{c}\text { Averaged emissions } \\
(\mathrm{kg} / \mathrm{d})\end{array}$ & Reported emissions \\
\hline \multirow{2}{*}{$\begin{array}{c}\text { Natural gas distribution } \\
\text { network }\end{array}$} & Von Fischer method & $190-1,370$ & - & - \\
\cline { 2 - 5 } & Weller method & $60-320$ & - & 6,900 \\
\hline \multirow{3}{*}{ Landfills } & Thackeray & $900-11,100$ & 2,600 & 58,700 \\
\cline { 2 - 5 } & Keele Valley & $600-11,200$ & 4,600 & - \\
\hline \multirow{3}{*}{ Gas compressor stations } & Parkway & $300-1,400$ & 600 & - \\
\cline { 2 - 5 } & Parkway (enhanced) & $3,600-4,000$ & 3,800 & - \\
\hline & Maple & $300-1,400$ & 800 & - \\
\hline
\end{tabular}

Table S2: Summary table of the emission rates estimated for different methane sources.

Figure S10: Map locating major sources of methane from downtown Toronto with the Gaussian Mixture Cluster Analysis. Enhancements of methane are represented by coloured open circles. Each cluster of open circles is differentiated by its colour. Red map marker icons with white question mark symbols represent the estimated location of methane sources and blue map marker icons with checkmarks identify the location of known sources.

\subsection{Summary table}

Table S2 summarizes the emission rates estimated for different methane sources in this study.

\subsection{Datasets used in the inverse modeling approach}

Table S3 provides information on the datasets used to estimate methane emissions for the different sites with the inverse modeling approach. 


\begin{tabular}{|c|c|c|c|c|c|c|}
\hline Analytical Platform (DOI) & Data File & $\begin{array}{l}\text { Keele Valley } \\
\text { Landfill }\end{array}$ & $\begin{array}{l}\text { Thackeray } \\
\text { Landfill }\end{array}$ & $\begin{array}{l}\text { Parkway } \\
\text { Compressor } \\
\text { Station }\end{array}$ & $\begin{array}{c}\text { Maple } \\
\text { Compressor } \\
\text { Station }\end{array}$ & $\begin{array}{l}\text { Keating } \\
\text { Channel }\end{array}$ \\
\hline $\begin{array}{c}\text { Bicycle } \\
\text { (https://doi.org/10.5683/SP/DEQJGQ) }\end{array}$ & sync_data_2018-06-28.csv & & $\mathrm{x}$ & & & \\
\hline Car & sync_data_2018-08-16.csv & $\mathrm{x}$ & & & & \\
\hline Car & sync_data_2018-08-30.csv & $\mathrm{x}$ & $x$ & & $x$ & \\
\hline Car & sync_data_2018-10-03.csv & & & $\mathrm{x}$ & $x$ & \\
\hline Car & sync_data_2018-10-06.csv & $x$ & $x$ & $x$ & $x$ & \\
\hline Car & sync_data_2018-10-13.csv & $x$ & & & & \\
\hline Car & sync_data_2018-11-28.csv & $\mathrm{x}$ & & & & \\
\hline Car & sync_data_2018-11-29.csv & $\mathrm{x}$ & $\mathrm{x}$ & & & \\
\hline Car & sync_data_2018-12-04.csv & & $x$ & & & \\
\hline Car & sync_data_2018-12-07.csv & & $x$ & & & \\
\hline Car & sync_data_2019-04-09.csv & $\mathrm{x}$ & $\mathrm{x}$ & $\mathrm{x}$ & & \\
\hline Car & sync_data_2019-04-15.csv & & $\mathrm{x}$ & $\mathrm{x}$ & & \\
\hline Car & sync_data_2019-04-25.csv & & $\mathrm{x}$ & & & \\
\hline $\begin{array}{c}\text { Bicycle } \\
\text { (https://doi.org/10.5683/SP2/SBIZ1F) }\end{array}$ & sync_data_2019-05-17.csv & & & & & $x$ \\
\hline $\begin{array}{c}\text { Bicycle } \\
\text { (https://doi.org/10.5683/SP2/SBIZ1F) }\end{array}$ & sync_data_2019-05-27.csv & & & & & $\mathrm{x}$ \\
\hline Car & sync_data_2019-06-05.csv & $\mathrm{x}$ & & & $x$ & \\
\hline $\begin{array}{c}\text { Bicycle } \\
\text { (https://doi.org/10.5683/SP2/SBIZ1F) }\end{array}$ & sync_data_2019-06-07.csv & & & & & $\mathrm{x}$ \\
\hline $\begin{array}{c}\text { Bicycle } \\
\text { (https://doi.org/10.5683/SP2/SBIZ1F) }\end{array}$ & sync_data_2019-06-19.csv & & & & & $\mathrm{x}$ \\
\hline Car & sync_data_2019-06-21.csv & & $\mathrm{x}$ & & & \\
\hline $\begin{array}{c}\text { Bicycle } \\
\text { (https://doi.org/10.5683/SP2/SBIZ1F) }\end{array}$ & sync_data_2019-07-03.csv & & & & & $\mathrm{x}$ \\
\hline $\begin{array}{c}\text { Bicycle } \\
\text { (https://doi.org/10.5683/SP2/SBIZ1F) }\end{array}$ & sync_data_2019-07-15.csv & & & & & $\mathrm{x}$ \\
\hline $\begin{array}{c}\text { Bicycle } \\
\text { (https://doi.org/10.5683/SP2/SBIZ1F) }\end{array}$ & sync_data_2019-07-29.csv & & & & & $\mathrm{x}$ \\
\hline
\end{tabular}

Table S3: List of the datasets used in the inverse modeling approach for the different sites.

\section{REFERENCES}

249 (1) Atherton, E., Risk, D., Fougère, C., Lavoie, M., Marshall, A., Werring, J., Williams, J. P., and

250 Minions, $C$.: Mobile measurement of methane emissions from natural gas developments in

251 northeastern British Columbia, Canada, Atmospheric Chemistry and Physics, 17, 12 405-12 420,

252 doi:https://doi.org/10. 5194/acp-17-12405-2017, URL https://www.atmos-chem-

253 phys.net/17/12405/2017/, 2017.

(2) O'Connell, E., Risk, D., Atherton, E., Bourlon, E., Fougère, C., Baillie, J., Lowry, D., and Johnson, J.: Methane emissions from contrasting production regions within Alberta, Canada: Implications under incoming federal methane regulations, Elem Sci Anth, 7, 3, doi:10.1525/elementa.341, URL http://www.elementascience.org/article/10.1525/elementa.341/, 2019.

(3) Ars, S., Broquet, G., Yver Kwok, C., Roustan, Y., Wu, L., Arzoumanian, E., and Bousquet, P.: Statistical atmospheric inversion of local gas emissions by coupling the tracer release technique and local-scale transport modelling: a test case with controlled methane emissions, Atmospheric Measurement Techniques, 10, 5017-5037, doi:https://doi.org/10.5194/amt-10-5017-2017, URL 
(4) Pedregosa, F., Varoquaux, G., Gramfort, A., Michel, V., Thirion, B., Grisel, O., Blondel, M., Prettenhofer, P., Weiss, R., Dubourg, V., Vanderplas, J., Passos, A., Cournapeau, D., Brucher, M.,

268 Perrot, M., and Duchesnay, : Scikit-learn: Machine Learning in Python, Journal of Machine Learning 269 Research, 12, 2825-2830, URL http://www.jmlr.org/papers/v12/pedregosa11a.html, 2011. 